\title{
An Algorithm for Spectrum Hole Detection using Convex Optimization And Tensor Analysis In Cognitive Radio Network
}

\author{
Emeshili O. Joseph ${ }^{1}$, Emmanuel Eronu ${ }^{2}$, Evans Ashigwuike ${ }^{3}$ \\ ${ }^{123}$ Department of Electrical/Electronics Engineering, University of Abuja Nigeria, \\ ofsyn@yahoo.com,majieronu2007@hotmail.com, ecashigwuike@ymail.com
}

\begin{abstract}
The issue of speed and accuracy is one major challenge in the area of Spectrum hole detection in Cognitive Radio Network(CRN), owing to some of the techniques used in the previous past, noise is sometimes recorded against spectrum hole, and this is mostly due to the method adopted, the need for a more compact procedure as become necessary. An Algorithm for Spectrum Hole Detecting using Convex Optimization and Tensor analysis in Cognitive Radio Network seeks to present a way out of it. The tensor analysis will provide an infinite representation Spectrum data from the wideband, while Convex optimization will help split the large data by grouping it into various spectrum segment, based on the objective function, this grouping will help improve on the speed of Spectrum hole detection. Principal Component Analysis(PCA) checks the level of correction using orthogonal transformation, the use of Eigen Values and Eigen Vectors will further help linearize the function by finding the roots. Covariance matrix will help further check how the variable varies together. It describes the dimension of the spectrum data. Diagonisation is used to extract the matrix with the spectrum data using singular value decomposition; finally, Bayesian inference will optimise decision making for spectrum data.
\end{abstract}

Keywords: Cognitive Radio, Tensor, Spectrum hole, Convex optimization, Covariance Matrix, Eigen Vector, Principal Component Analysis

\section{Introduction}

Reliable and fast wireless data transmission is becoming a global phenomenon and also a significant consideration in our lives, such as the internet, online shopping, and social networking. This has caused an increase in the demand for the radio frequency spectrum. However, conservative spectrum allocation policies have created a shortage of vacant spectrum bands (channels) FCC. The US frequency allocation given in indicates that there is little room for any new assignment in the most useful frequency bands $(<3 \mathrm{GHz})$ for wireless communications.

On the other hand, recent studies conducted by the Federal Communications Commission (FCC) in the United States and Ofcom in the United Kingdom [3] have found that the average utilization in licensed frequency bands is as low as $5 \%$. This is also indicated by the spectrum measurements carried out in our laboratory within a range of $1 \mathrm{MHz}$ to $1 \mathrm{GHz}$, which show large swathes of the inactive spectrum (Figure. 1). Based on these measurements, the FCC concluded that there are two basic scenarios to improve the 
spectrum efficiency of the licensed bands. In the first scenario, where the spectrum is fully utilised, the spectrum efficiency can be improved in terms of bits per second per Hertz (bps/Hz), by using better radio access technologies. In Spectrum Prediction in Cognitive Radio Networks: A Bayesian Approach [1]

Given this fact, new insights into the use of radio spectrum have challenged the traditional static spectrum allocation policy. Actual measurements have revealed that most of the allocated spectrum is mostly underutilized. Similar views on the underutilization of allocated spectrum were reported by the SpectrumPolicy Task Force appointed by Federal Communications Commission's (FCC) Spectral efficiency can be increased significantly by giving opportunistic access of the frequency bands to unlicensed users. Cognitive Radio (CR) has been proposed as a way to improve spectrum efficiency by exploiting the unused spectrum in dynamically changing environments. The CR technology is based on an innovative radio design philosophy which involves smartly sensing the swaths of radio spectrum and then determining the transmission characteristics (e.g., symbol rate, power, bandwidth) for cognitive radio users (CR up until recently, most research has been concentrated on improving spectrum efficiency in bps/Hz by practising better radio access technologies.

Cognitive Radio (CR) is an emerging technique to increase the practical usage of the spectrum. Due to the rapid development in some wireless devices and gadgets, there is a scarcity in the radio frequency band. This led to the spectrum sensing concept of identifying the unused spectrum holes. Spectrum sensing can be defined as the ability to detect the presence or absence of a licensed user in the channel, while the most challenging task is to sense in shallow SNR regime [2]

In current communication networks, the average spectrum utilization is between $15 \%$ to $85 \%$. Cognitive Radio (CR) is a solution to increase the spectrum utilization and ultimately, the network capacity leading to generating new revenue streams with a higher quality of service. With the growing demand for higher capacity in wireless networks due to the rapid growth of new applications such as multimedia, the network resources such as spectrum should be used more efficiently to fulfil the need for both quantity and quality of service.

\subsection{Cognitive Radio: Approach to Increase Spectrum Efficiency}

One major finding from the measurements reported is that a large portion of the radio spectrum is not in use for significant periods in certain areas. Hence, there are many spectrum holes. It is defined as a set of frequency bands assigned (licensed) to a user (this user is referred to as a primary user), but, at a particular time and a specific geographic location, the frequency band is not being used by that user.

Moreover, the report also showed that users heavily access most of the unlicensed spectra and therefore have a high spectrum utilization thanks to the possibility of open access with relaxed regulations. These observations lead us to a critical idea: the spectrum utilization can be drastically increased by allowing secondary users access to the spectrum holes that are unutilized by the primary user at a certain time and space. The Cognitive radio has been proposed as a means to achieve such dynamics. A cognitive radio senses the spectral environment over a full frequency band and exploits this information to opportunistically provide wireless links that can best meet the demand of the user, but also of its radio environments.

CR facilitates the secondary user (SU) to build transmission links in unoccupied PU channels such that there is no/minimum interference to PUs. The CR possesses the following functionalities: spectrum sensing; spectrum access; spectrum allocation and management among different SUs. The SU's 
Emeshili O. Joseph, Emmanuel Eronu, Evans Ashigwuike; An Algorithm for Spectrum Hole Detection using Convex Optimization And Tensor Analysis In Cognitive Radio Network. Journal of Biomedical Engineering and Medical Imaging, Volume 6, No 6, December (2019), pp 1-24

transmission modes can be generally categorized into two modes: interference avoidance (white space) mode and the Interference management (black and grey space) mode. The interference avoidance mode is often termed as interweaving access. In interweave access, the SU finds spectrum holes (white space) by sensing the radio frequency spectrum as illustrated in figure 1 [1]

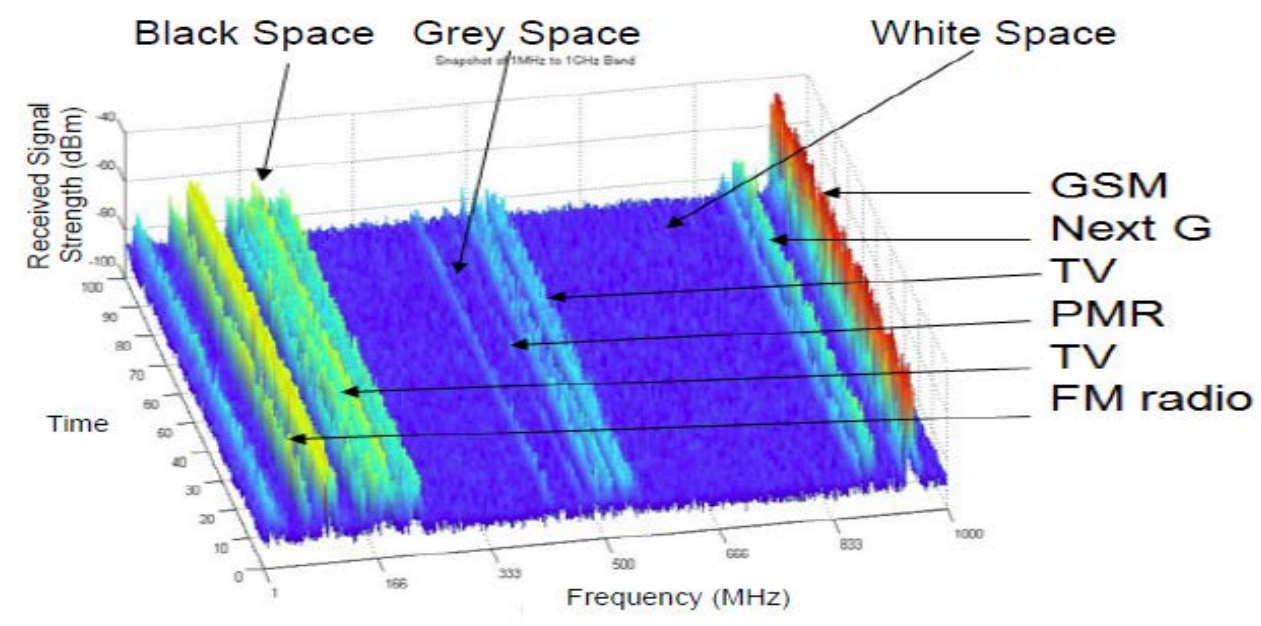

Figure 1: Spectrum measurements. [4]

One of the most critical discoveries from the measurements reported is that a large portion of the scenario, where the spectrum usage is relatively low over time, the spectrum efficiency can be improved by increasing the access efficiency, i.e. allowing access of unlicensed (secondary) devices to the licensed (primary) frequency bands. Up until recently, most research has been concentrated on improving spectrum efficiency in bps/Hz. There has, however, been an increase in research effort directed towards increasing access efficiency through spectrum sharing. One of the critical enabling technologies in this push is cognitive radio (CR), which. CR enables the secondary user ( $\mathrm{SU}$ ) to build transmission links in vacant $\mathrm{PU}$ channels such that there is no/minimum interference to PUs.

The realisation of such an operation requires the $C R$ to have the following functionalities: spectrum sensing; spectrum access; spectrum allocation and management among different SUs; and reconfigurable hardware.

\subsection{Speed and Accuracy in Spectrum Sensing}

Wideband spectrum sensing is one of the most challenging components of cognitive radio networks. It should be performed as fast and accurately as possible. Speed is also a significant concern in cognitive radio, [8]

Since it takes split seconds for reallocation based on the availability of several Secondary users in the queue. Some of the most essential elements of the cognitive radio concept is its ability to measure, sense, learn, and know the parameters associated with the radio channel characteristics, the availability of spectrum and power, the radio's operating environment, user requirements and applications, available network infrastructures) and nodes, local policies and other operating restrictions. In cognitive radio terminology, the primary users are defined as the users who have higher priority or legacy rights on the 
usage of a specific part of the spectrum. On the other hand, secondary users, which have a lower priority, utilise this spectrum in such a way that they do not cause interference to primary users. Therefore, secondary users need to have cognitive radio capabilities, such as sensing the spectrum reliably to check whether a primary user is using it and to change the radio parameters to exploit the remaining part of the range.

Speed can be enhanced when priorities are set, and objective five functions are spelt out[9]

This channel is also used by the primary users and must be vacated immediately when the primary users arrive. Due to the nature of cognitive radio, the operating frequency change continuously; this is referred to as a dynamic radio range property. The research on spectrum sharing techniques considers the channel as a basic spectrum unit. In many of the research about spectrum sharing, researchers found the assumption that the secondary users know the location and transmission power of the primary users to calculate the interferences appropriately.

\subsection{Cognitive Radio}

Cognitive Radio is considered as a solution to overcome the spectrum scarcity in the present communication scenario. Spectrum sensing is a significant activity that is used by the CR to understand the spectrum occupancy of PU. Several methods are proposed in the literature to sense the spectrum hole. A CR user develops a spectrum pool consisting of all the spectrum holes in a range of spectrum and chooses the optimum one for its future usage. Channel capacity can be increased by using the proper spectrum sharing policy. CR users are supposed to operate within tiny time slots for spectrum sensing and to communicate with other users. Spectrum sensing, spectrum decision and spectrum sharing will take considerable time delays. If it takes more time for these activities, then the time available for data communication will be less, and the throughput of the system will also come down. Spectrum prediction will be an alternate approach to save sensing time. Spectrum prediction in cognitive radio networks is a challenging problem that involves several sub-topics such as channel status prediction, PU activity prediction, radio environment prediction, and transmission rate prediction. Prediction based spectrum sensing, prediction based spectrum decision and prediction based spectrum mobility have been presented in the literature. Prediction based sensing is explored in this paper. In this case, the channel predicted to be busy can be omitted by the SU form sensing so that there will be time-saving and energy saving. In CR networks, since SUs are sensing and observing the spectrum all the time, they can learn the usage pattern of the spectrum and use such information to predict the future status of the spectrum. [1]

Spectrum Sensing Model Spectrum sensing is one of the essential processes performed by cognitive radio systems. It allows the SUs to learn about the radio environment by detecting the presence of the PU signals using one or multiple techniques and decide to transmit or not in its frequency band, as shown in figure 2. Where $n=1 \ldots N, N$ is the number of samples, $y(n)$ is the SU received signal, $s(n)$ is the PU signal, $w(n)$ is the additive white Gaussian noise (AWGN) with zero mean and variance, and $\mathrm{h}$ is the complex channel gain of the sensing channel. $\mathrm{HO}$ and $\mathrm{H} 1$ denote the absence and the presence of the PU signal, respectively. The PU signal detection is performed using one of the spectrum sensing techniques to decide between the two hypotheses $\mathrm{HO}$ and $\mathrm{H} 1$. The detector output, also called the test statistic, is then compared to a threshold to make the sensing decision about the PU signal presence. The sensing decision is performed as [3][4] 
Emeshili O. Joseph, Emmanuel Eronu, Evans Ashigwuike; An Algorithm for Spectrum Hole Detection using Convex Optimization And Tensor Analysis In Cognitive Radio Network. Journal of Biomedical Engineering and Medical Imaging, Volume 6, No 6, December (2019), pp 1-24

$$
y(n)= \begin{cases}w(n), & H_{0}: P U \text { absent } \\ h * s(n)+w(n), & H_{1}: P U \text { Present }\end{cases}
$$

Where $n=1 \ldots . N, N$ is the number of samples, $y(n)$ is the SU received signal, $s(n)$ is the PU signal, $w(n)$ is the additive white Gaussian noise (AWGN) with zero mean and variance, and $\mathrm{h}$ is the complex channel gain of the sensing channel. $\mathrm{HO}$ and $\mathrm{H} 1$ denote the absence and the presence of the PU signal, respectively. The $\mathrm{PU}$ signal detection is performed using one of the spectrum sensing techniques to decide between the two hypotheses $\mathrm{H}_{0}$ and $\mathrm{H}_{1}$.

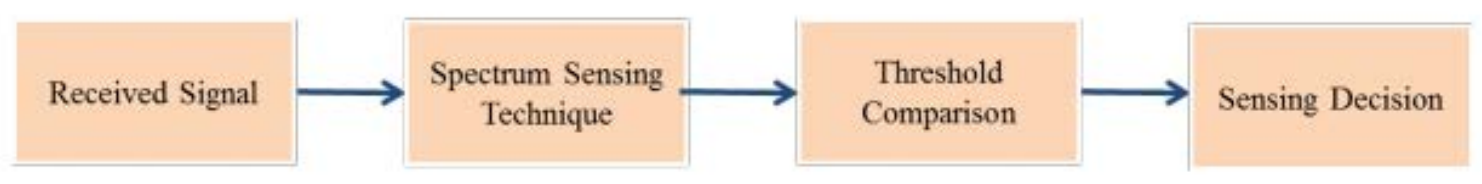

Figure 2 Block Diagram Flow Process for CR

\section{Tensor}

Tensor is a multi-way extension of a matrix, and Tensor A multi-dimensional array will be used to decompose frequency and also model the system for accuracy, tensors are geometric objects which illustrate the linear relations between geometric vectors, scalars, and other tensors. Simple examples of such relationships are the dot product, the cross product, and the linear maps. The Geometric vectors, often used in physics and engineering applications, and scalars themselves are also tensors. Figure 3 and 4 are a more sophisticated example is the Cauchy stress tensor $T$, which has a direction $v$ as input and then produces the stress $T(v)$ on the surface normal to this vector as the output, thus expressing a relationship between these two vectors, shown in the figure 3 below. Let denote unit vectors in the $x, y$ and z-direction. The hat indicates a magnitude of unity

The position vector (the arrow denotes a vector that is not a unit vector) is given as

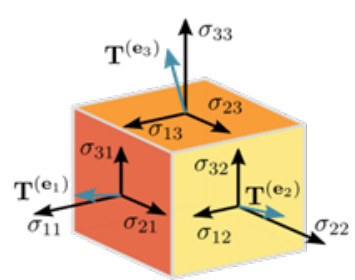

Figure 3 3D Tensor diagram

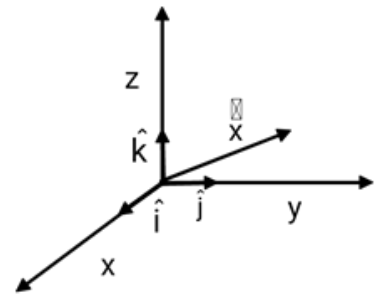

Figure 4 3D Vector Diagram

Taking a look at how tensor can be represented, an example is positive normal stress puts a body in tension, and negative normal stress puts the body in compression. Shear stresses always put the body in shear.

An instance can be considered for tensor differential if $x^{i}$ is the coordinate of a point in $n$-dimensional space $d x^{i}$ are component of a contravariant vector. If $x^{1}, x^{2}, \ldots, x^{n}$ or $x^{i}$ are coordinates in $X$ coordinate system and $\underline{x}^{1}, \underline{x}^{2}, \ldots, \underline{x}^{n}$, or $\underline{x}^{i}$ are coordinate in $Y$-coordinate system. 


$$
\begin{array}{r}
\text { If } \underline{x}^{i}=\underline{x}^{i}\left(x^{1}, x^{2}, \ldots, x^{n}\right) \\
d \underline{x}^{i}=\frac{\partial \underline{x}^{i}}{\partial x^{1}} d x^{1}+\frac{\partial \underline{x}^{i}}{\partial x^{2}} d x^{2}+\cdots+\frac{\partial \underline{x}^{i}}{\partial x^{n}} d x^{n} \\
d \underline{x}^{i}=\frac{\partial \underline{x}^{i}}{\partial x^{j}} d x^{j}
\end{array}
$$

It is the law of transformation of the contravariant vector. So $d x^{i}$ are components of a contravariant vector.

If $\frac{\partial \emptyset}{\partial x^{i}}$ is a covariant vector where $\emptyset$ is a scalar function.

Then $x^{1}, x^{2}, \ldots, x^{n}$ or $x^{i}$ are coordinates in $X$-coordinate system and $\underline{x}^{1}, \underline{x}^{2}, \ldots, \underline{x}^{n}$ or $\underline{x}^{i}$ are coordinates in $Y$-coordinate system.

$$
\text { Consider } \begin{aligned}
\emptyset\left(\underline{x}^{1}, \underline{x}^{2}, \ldots, \underline{x}^{n}\right) & =\emptyset\left(x^{1}, x^{2}, \ldots, x^{n}\right) \\
\partial \emptyset & =\frac{\partial \emptyset}{\partial x^{1}} \partial x^{1}+\frac{\partial \emptyset}{\partial x^{2}} \partial x^{2}+\cdots+\frac{\partial \emptyset}{\partial x^{n}} \partial x^{n} \\
\frac{\partial \emptyset}{\partial \underline{x}^{i}} & =\frac{\partial \emptyset}{\partial x^{1}} \frac{\partial x^{1}}{\partial \underline{x}^{i}}+\frac{\partial \emptyset}{\partial x^{2}} \frac{\partial x^{2}}{\partial \underline{x}^{i}}+\cdots+\frac{\partial \emptyset}{\partial x^{n}} \frac{\partial x^{n}}{\partial \underline{x}^{i}} \\
\frac{\partial \emptyset}{\partial \underline{x}^{i}} & =\frac{\partial \emptyset}{\partial x^{j}} \frac{\partial x^{j}}{\partial \underline{x}^{i}} \quad \text { or } \quad \frac{\partial \emptyset}{\partial \underline{x}^{i}}=\frac{\partial x^{j}}{\partial \underline{x}^{i}} \frac{\partial \emptyset}{\partial x^{j}}
\end{aligned}
$$

It is the law of transformation of the component of the covariant vector. So $\frac{\partial \emptyset}{\partial x^{i}}$ is a component of the covariant vector

\subsection{Covariant Tensor of Rank Two}

For this instance Let $A_{i j}(i, j=1,2, \ldots, n)$ be $n^{2}$ functions of coordinates $x^{1}, x^{2}, \ldots, x^{n}$ in $X$-coordinate system. If the quantities $A_{i j}$ are transformed to $A_{i j}$ in $Y$-coordinate system having coordinates $\underline{x}^{1}, \underline{x}^{2}, \ldots, \underline{x}^{n}$, then according to the law of transformation,

$$
\underline{A_{i j}}=\frac{\partial x^{k}}{\partial \underline{x}^{i}} \frac{\partial x^{1}}{\partial \underline{x}^{j}} A_{k 1}
$$

Then $A_{i j}$ called components of the covariant tensor of rank two

\section{Procedure Taken for the Research}

This research will employ Mathematical modelling, and Software design approaches, The algorithm for realising this work will follow the under listed order of fundamental is the order of realisation of this work.
1. Tensor
2. Convex optimization
2. Principal Component Analysis
3. Eigen Values and Eigen Vectors
4. Covariant Matrix
5. Singular Value Decomposition
6. Bayesian Inference 
Emeshili O. Joseph, Emmanuel Eronu, Evans Ashigwuike; An Algorithm for Spectrum Hole Detection using Convex Optimization And Tensor Analysis In Cognitive Radio Network. Journal of Biomedical Engineering and Medical Imaging, Volume 6, No 6, December (2019), pp $1-24$

\subsection{Splitting Frequency}

Wideband and Ultra-wideband have wide range of spectrum, spectrum data will be a daunting task to measure, accuracy and time is an issue for concern, owing to the possibility of a large number of data samples in the terminal sub-band channels of the wideband spectrum, developing a fast and accurate spectrum detecting technique is very important, tensor analysis will be useful for representing these massive quantities of data before detection. Due to the extensive data associated wideband, different levels of decomposition is necessary.

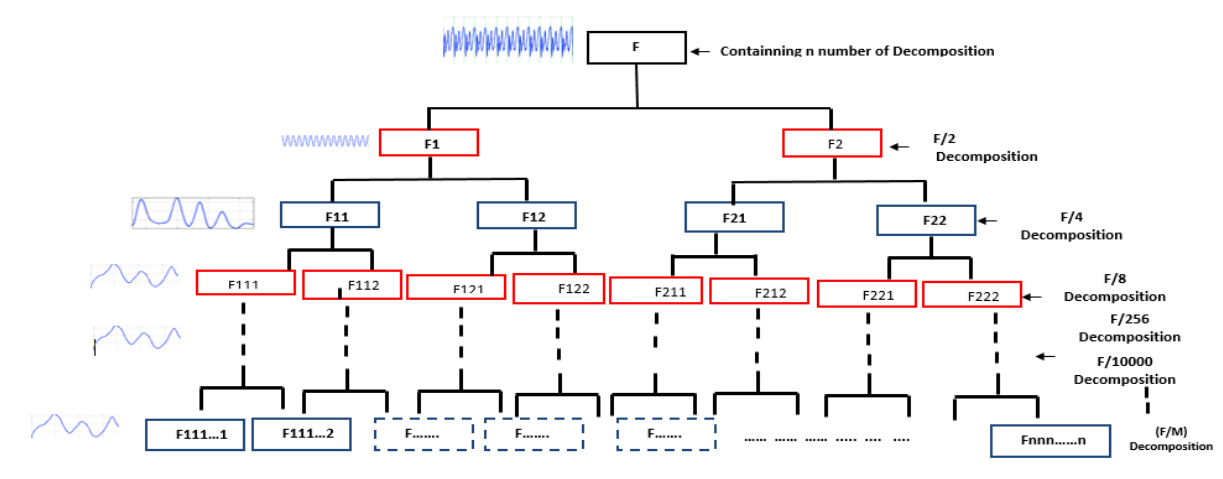

Figure 5 Top-Down Frequency Decomposition

The diagram in figure 5 is a top-down arrangement that shows how a spectrum can be split into a different range of frequencies to accommodate the wide range of spectrum within a wideband. While figure 6 is an analogy showing how wideband can be split into different sub-bands

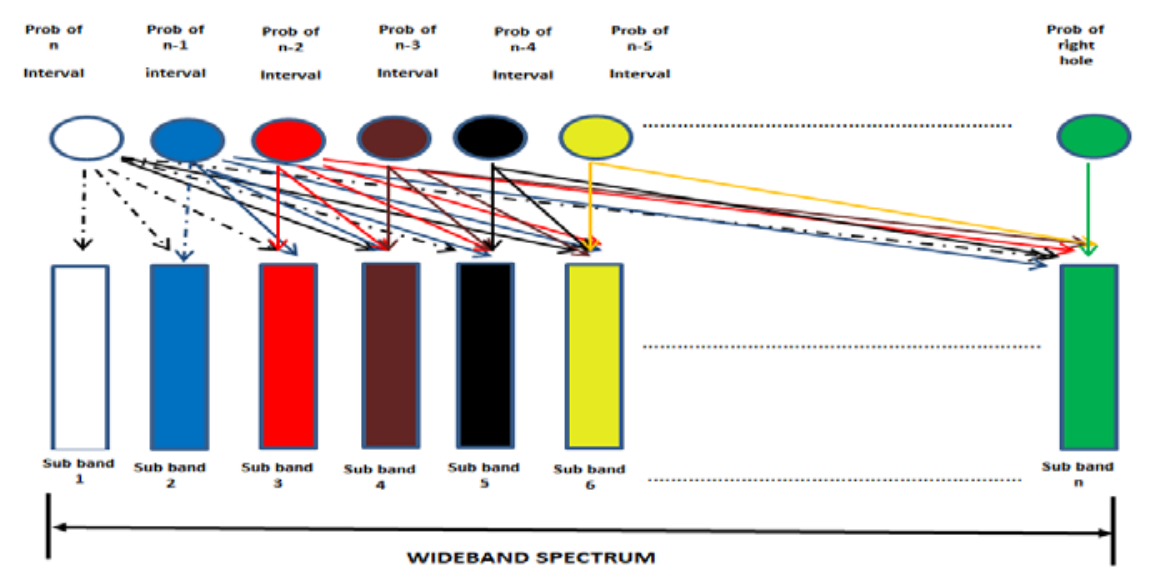

Figure 6 diagram showing analogy of wideband is broken down to many sub-bands,

In representing the frequency spread of the wideband, this work adopts the properties of the tensors in tagging frequency position against energy as its usually represented in Power Spectrum Density (PSD), which can handle an infinite number of representation.

\subsection{Power Spectral Density}

Power Spectral Density (PSD), is defined as the frequency response of a random or periodic signal. It shows where the average power is distributed as a function of frequency. 
The PSD is deterministic, and for certain types of random signals, it is independent of time ${ }^{1}$. This is very useful because the Fourier transform of a random time signal is itself random, and therefore is of little use calculating the transfer relationships (i.e., determining the output of a filter when its input is random). The PSD of a random time signal, given as $\mathrm{x}(\mathrm{t})$ can be expressed in one of two methods that are equivalent to each other

1. The PSD is the average of the Fourier transform's magnitude squared, over an extensive time interval

$$
S_{x}(f)=\lim _{T \rightarrow \infty} E\left\{\frac{\mathbf{1}}{2 T}\left|\int_{-T}^{T} x(t) e^{-j 2 \pi f t} d t\right|^{2}\right\}
$$

2. The PSD is the Fourier transform of the autocorrelation function.

$$
\begin{aligned}
& S_{x}(f)=\int_{-T}^{T} R_{x}(\tau) e^{-j 2 \pi f t} d t \\
& R_{x}(\tau)=E\left\{X(t) X^{*}(t+\tau)\right\}
\end{aligned}
$$

- The power can be calculated from a random signal over a given band of frequencies as follows:

1. Total Power in $\mathrm{x}(\mathrm{t})$ :

$$
P=\int_{-\infty}^{\infty} S_{X}(f) d f=R_{X}(\mathbf{0})
$$

2. Power in $x(t)$ in range $f_{1}-f_{2}$ :

$$
P_{12}=\int_{f_{1}}^{f_{2}} S_{x}(f) d f=R_{x}(\mathbf{0})
$$

$$
(f)=S_{x}(f)|H(f)|^{2}
$$

This power spectral density (PSD), or simply the power spectrum, of a signal, is the representation of the spread of signal power as a function of frequency. Power Spectrum Density is used to measure the signal level of the spectrum data; the spectrum data will be represented on the various tensor slice.

\subsection{Preliminary Data}

Preliminary data was gotten using Periodogram simulation lab in Matlab, and it was used to generate data, as displayed by graph in figure 7, 8 and 9, these are Periodogram used to represent frequency plot of energy level against frequency. Power spectral density is used in identifying the approximate location of the spectrum hole. This will be improved upon with the help of the thresholding technique to get the exact location

Figures 7, 8 and 9 are the graphs of wideband signals, and the figure has protruded point which indicates the area where the highest energy level, the points will be further decomposed and split to help simplify the process. Note figure 10 is a plot showing thresholding of a sub-band. 
Emeshili O. Joseph, Emmanuel Eronu, Evans Ashigwuike; An Algorithm for Spectrum Hole Detection using Convex Optimization And Tensor Analysis In Cognitive Radio Network. Journal of Biomedical Engineering and Medical Imaging, Volume 6, No 6, December (2019), pp 1-24

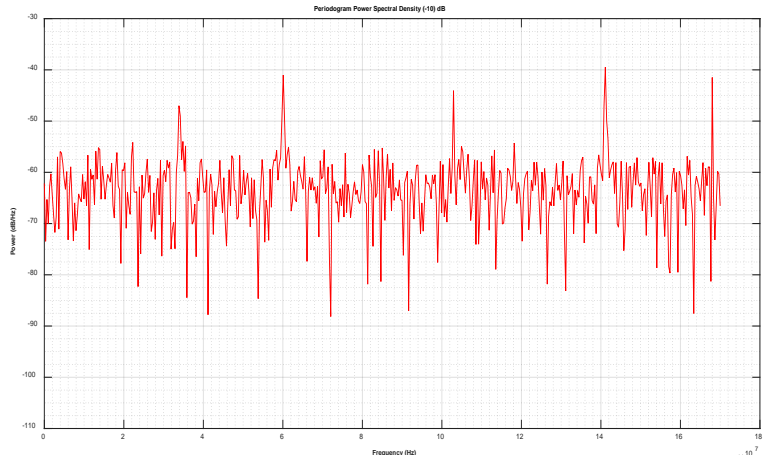

Figure 7 Graph of Periodogram Power spectral density 1

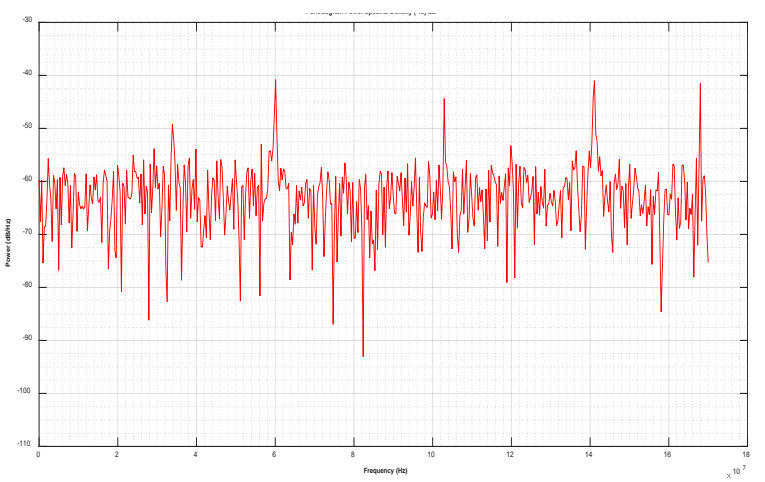

Figure 9 Periodogram Power spectral density 3

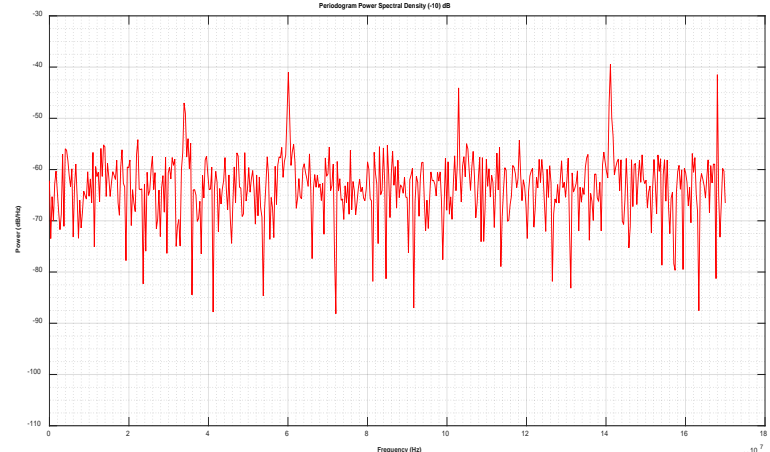

Figure 8 Periodogram Power spectral density 2

18

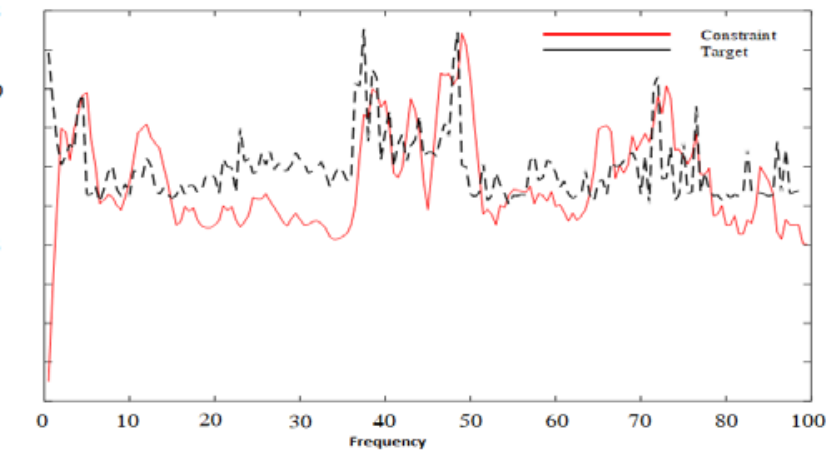

Figure 10 Periodogram Power spectral density with constraint 1 
Table 1 Frequency against PSD

\begin{tabular}{|c|c|c|c|c|c|c|c|c|c|c|c|}
\hline Frequency & Power & Frequency & Power & Frequency & Power & Frequency & Power & Frequency & Power & Frequency & Power \\
\hline 0.0033 & $\begin{array}{l}-68.0124 \\
\end{array}$ & 0.2191 & $\begin{array}{l}-64.6777 \\
\end{array}$ & 0.4350 & -58.6489 & 0.6508 & -54.7599 & 0.8633 & -64.3586 & 1.0758 & -72.9718 \\
\hline 0.0066 & -63.7203 & 0.2225 & -57.7271 & 0.4383 & -63.5167 & 0.6541 & -62.0849 & 0.8666 & -67.5175 & 1.0791 & -60.9877 \\
\hline 0.0100 & -63.3896 & 0.2258 & -59.4798 & 0.4416 & -63.1784 & 0.6574 & -69.0151 & 0.8699 & -70.8929 & 1.0824 & -62.3058 \\
\hline 0.0133 & -59.7945 & 0.2291 & -63.3936 & 0.4449 & -59.6923 & 0.6607 & -59.7298 & 0.8732 & -62.6825 & 1.0857 & -60.1250 \\
\hline 0.0166 & -70.6357 & 0.2324 & -71.2143 & 0.4482 & -63.5276 & 0.6641 & -66.6766 & 0.8766 & -65.2664 & 1.0891 & -71.4327 \\
\hline 0.0199 & -62.6848 & 0.2357 & -57.6382 & 0.4516 & -60.4034 & 0.6674 & -61.9197 & 0.8799 & -62.5539 & 1.0924 & -82.0991 \\
\hline 0.0232 & -64.6324 & 0.2391 & -62.7363 & 0.4549 & -59.3305 & 0.6707 & -62.8936 & 0.8832 & -62.7829 & 1.0957 & -65.9897 \\
\hline 0.0266 & -67.8683 & 0.2424 & -79.5291 & 0.4582 & -71.2839 & 0.6740 & -60.0756 & 0.8865 & -58.2590 & 1.0990 & -62.2241 \\
\hline 0.0299 & -54.6270 & 0.2457 & -60.2172 & 0.4615 & -70.8024 & 0.6773 & -74.8513 & 0.8898 & -63.8885 & 1.1023 & -72.7133 \\
\hline 0.0332 & -61.4575 & 0.2490 & -64.9404 & 0.4648 & -55.4131 & 0.6807 & -66.1512 & 0.8932 & -57.4035 & 1.1057 & -64.1454 \\
\hline 0.0365 & -60.8351 & 0.2523 & -65.2638 & 0.4682 & -75.8376 & 0.6840 & -63.3449 & 0.8965 & -63.9255 & 1.1090 & -61.5283 \\
\hline 0.0398 & -60.5575 & 0.2557 & -57.3546 & 0.4715 & -66.1869 & 0.6873 & -62.4482 & 0.8998 & -60.2164 & 1.1123 & -61.0063 \\
\hline 0.0432 & -63.8667 & 0.2590 & -68.5535 & 0.4748 & -58.6427 & 0.6906 & -63.1162 & 0.9031 & -61.8184 & 1.1156 & -58.7389 \\
\hline 0.0465 & -66.4383 & 0.2623 & -56.3661 & 0.4781 & -59.9192 & 0.6939 & -58.4851 & 0.9064 & -59.2813 & 1.1189 & -61.4926 \\
\hline 0.0498 & -69.7666 & 0.2656 & -56.1146 & 0.4814 & -75.3531 & 0.6973 & -73.7983 & 0.9098 & -61.8391 & 1.1223 & -67.3417 \\
\hline 0.0531 & -63.2633 & 0.2689 & -65.4437 & 0.4848 & -60.1404 & 0.7006 & -57.6075 & 0.9131 & -57.9839 & 1.1256 & -58.1062 \\
\hline 0.0564 & -60.2411 & 0.2723 & -66.8126 & 0.4881 & -58.8223 & 0.7039 & -64.7007 & 0.9164 & -71.2326 & 1.1289 & -58.6922 \\
\hline 0.0598 & -62.3088 & 0.2756 & -63.4329 & 0.4914 & -66.8632 & 0.7072 & -65.4389 & 0.9197 & -61.7776 & 1.1322 & -78.7460 \\
\hline 0.0631 & -56.5846 & 0.2789 & -60.4598 & 0.4947 & -56.8382 & 0.7105 & -60.7913 & 0.9230 & -62.2408 & 1.1355 & -57.6501 \\
\hline 0.0664 & -68.2973 & 0.2822 & -63.6830 & 0.4980 & -64.0037 & 0.7139 & -62.0475 & 0.9264 & -59.2225 & 1.1389 & -63.9622 \\
\hline 0.0697 & -65.2242 & 0.2855 & -68.2009 & 0.5014 & -73.3670 & 0.7172 & -63.0583 & 0.9297 & -63.8286 & 1.1422 & -59.7888 \\
\hline 0.0730 & -57.4201 & 0.2889 & -75.2738 & 0.5047 & -61.5093 & 0.7205 & -61.8235 & 0.9330 & -61.6746 & 1.1455 & -66.7986 \\
\hline 0.0764 & -76.0218 & 0.2922 & -56.2375 & 0.5080 & -67.0520 & 0.7238 & -64.7633 & 0.9363 & -68.4276 & 1.1488 & -65.6203 \\
\hline 0.0797 & -56.5606 & 0.2955 & -59.4837 & 0.5113 & -59.8618 & 0.7271 & -62.1087 & 0.9396 & -63.9500 & 1.1521 & -56.9194 \\
\hline 0.0830 & -71.8076 & 0.2988 & -57.7663 & 0.5146 & -54.7398 & 0.7305 & -61.9486 & 0.9430 & -60.6760 & 1.1555 & -59.8322 \\
\hline 0.0863 & -59.1226 & 0.3021 & -60.8057 & 0.5180 & -60.3426 & 0.7338 & -57.9910 & 0.9463 & -64.0969 & 1.1588 & -64.8635 \\
\hline 0.0896 & -60.2307 & 0.3055 & -65.3100 & 0.5213 & -70.1913 & 0.7371 & -66.4739 & 0.9496 & -67.9528 & 1.1621 & -74.4397 \\
\hline 0.0930 & -63.9425 & 0.3088 & -59.1619 & 0.5246 & -59.0398 & 0.7404 & -61.7862 & 0.9529 & -58.1070 & 1.1654 & -56.8542 \\
\hline 0.0963 & -58.5296 & 0.3121 & -72.0362 & 0.5279 & -64.5315 & 0.7438 & -62.4410 & 0.9563 & -74.7542 & 1.1688 & -71.5921 \\
\hline 0.0996 & -61.0405 & 0.3154 & -68.0809 & 0.5313 & -58.1558 & 0.7471 & -72.9678 & 0.9596 & -68.3077 & 1.1721 & -71.1076 \\
\hline 0.1029 & -70.3289 & 0.3187 & -66.0693 & 0.5346 & -63.0071 & 0.7504 & -64.6985 & 0.9629 & -59.0771 & 1.1754 & -62.8709 \\
\hline 0.1063 & -63.2537 & 0.3221 & -58.1231 & 0.5379 & -59.3460 & 0.7537 & -66.7556 & 0.9662 & -61.9220 & 1.1787 & -64.1743 \\
\hline 0.1096 & -58.7235 & 0.3254 & -60.2765 & 0.5412 & -56.0338 & 0.7570 & -70.3988 & 0.9695 & -59.7458 & 1.1820 & -57.6795 \\
\hline 0.1129 & -61.1657 & 0.3287 & -57.4853 & 0.5445 & -63.7075 & 0.7604 & -68.9479 & 0.9729 & -68.0597 & 1.1854 & -58.9483 \\
\hline 0.1162 & -63.7523 & 0.3320 & -55.3816 & 0.5479 & -58.2717 & 0.7637 & -66.7712 & 0.9762 & -69.3697 & 1.1887 & -66.2912 \\
\hline 0.1195 & -64.8368 & 0.3354 & -57.6197 & 0.5512 & -63.1613 & 0.7670 & -64.9808 & 0.9795 & -67.0716 & 1.1920 & -60.9561 \\
\hline 0.1229 & -67.1263 & 0.3387 & -55.2362 & 0.5545 & -71.4085 & 0.7703 & -60.7439 & 0.9828 & -62.1931 & 1.1953 & -65.9365 \\
\hline 0.1262 & -74.3089 & 0.3420 & -49.1054 & 0.5578 & -69.8794 & 0.7736 & -60.6472 & 0.9861 & -59.6382 & 1.1986 & -82.1751 \\
\hline 0.1295 & -63.5413 & 0.3453 & -52.8879 & 0.5611 & -63.0006 & 0.7770 & -63.8818 & 0.9895 & -70.0904 & 1.2020 & -59.0900 \\
\hline 0.1328 & -58.5854 & 0.3486 & -59.9341 & 0.5645 & -55.2003 & 0.7803 & -60.4352 & 0.9928 & -67.7038 & 1.2053 & -61.0498 \\
\hline 0.1361 & -60.9775 & 0.3520 & -68.1865 & 0.5678 & -56.0437 & 0.7836 & -66.0002 & 0.9961 & -61.2145 & 1.2086 & -57.4254 \\
\hline 0.1395 & -60.1377 & 0.3553 & -62.7150 & 0.5711 & -62.5005 & 0.7869 & -66.0506 & 0.9994 & -63.4884 & 1.2119 & -59.3106 \\
\hline 0.1428 & -58.2753 & 0.3586 & -60.5955 & 0.5744 & -57.6602 & 0.7902 & -57.9623 & 1.0027 & -57.8645 & 1.2152 & -67.4526 \\
\hline 0.1461 & -65.1592 & 0.3619 & -60.5362 & 0.5777 & -70.8719 & 0.7936 & -64.7242 & 1.0061 & -57.6306 & 1.2186 & -58.2108 \\
\hline 0.1494 & -63.2326 & 0.3652 & -54.7313 & 0.5811 & -69.7574 & 0.7969 & -68.7141 & 1.0094 & -66.7457 & 1.2219 & -65.7881 \\
\hline 0.1527 & -57.0811 & 0.3686 & -66.6511 & 0.5844 & -62.6192 & 0.8002 & -64.9666 & 1.0127 & -60.6668 & 1.2252 & -58.4864 \\
\hline 0.1561 & -59.7747 & 0.3719 & -58.4703 & 0.5877 & -58.0100 & 0.8035 & -67.5324 & 1.0160 & -62.1234 & 1.2285 & -66.4000 \\
\hline 0.1594 & -64.3464 & 0.3752 & -62.7369 & 0.5910 & -56.8321 & 0.8068 & -61.1417 & 1.0193 & -62.8264 & 1.2318 & -65.7744 \\
\hline 0.1627 & -66.3173 & 0.3785 & -62.2333 & 0.5943 & -58.9874 & 0.8102 & -66.9222 & 1.0227 & -60.9429 & 1.2352 & -63.6673 \\
\hline 0.1660 & -75.3411 & 0.3818 & -60.7257 & 0.5977 & -60.2209 & 0.8135 & -61.4947 & 1.0260 & -56.9078 & 1.2385 & -60.9447 \\
\hline 0.1693 & -57.0930 & 0.3852 & -68.2646 & 0.6010 & -47.7033 & 0.8168 & -56.1715 & 1.0293 & -44.9638 & 1.2418 & -60.0327 \\
\hline 0.1727 & -63.0944 & 0.3885 & -62.8774 & 0.6043 & -39.7459 & 0.8201 & -64.5166 & 1.0326 & -59.1152 & 1.2451 & -63.6701 \\
\hline 0.1760 & -59.5607 & 0.3918 & -61.8436 & 0.6076 & -52.4537 & 0.8234 & -72.0578 & 1.0359 & -56.8783 & 1.2484 & -61.8175 \\
\hline 0.1793 & -72.9259 & 0.3951 & -62.7692 & 0.6109 & -53.9625 & 0.8268 & -57.1570 & 1.0393 & -58.2315 & 1.2518 & -59.1733 \\
\hline 0.1826 & -57.8639 & 0.3984 & -63.9667 & 0.6143 & -61.2897 & 0.8301 & -64.8675 & 1.0426 & -62.0727 & 1.2551 & -56.1840 \\
\hline 0.1859 & -65.3424 & 0.4018 & -62.9822 & 0.6176 & -61.9293 & 0.8334 & -60.0820 & 1.0459 & -64.3671 & 1.2584 & -60.5183 \\
\hline 0.1893 & -57.4137 & 0.4051 & -62.9607 & 0.6209 & -60.9614 & 0.8367 & -63.6253 & 1.0492 & -58.5105 & 1.2617 & -64.0376 \\
\hline 0.1926 & -60.6974 & 0.4084 & -72.0461 & 0.6242 & -66.6353 & 0.8400 & -61.5750 & 1.0525 & -61.2156 & 1.2650 & -70.0883 \\
\hline 0.1959 & -64.5482 & 0.4117 & -66.8793 & 0.6275 & -62.8998 & 0.8434 & -63.0807 & 1.0559 & -65.7243 & 1.2684 & -65.3084 \\
\hline 0.1992 & -77.0964 & 0.4150 & -82.1818 & 0.6309 & -60.69966 & 0.8467 & -63.6845 & 1.0592 & -61.9880 & 1.2717 & -62.2863 \\
\hline 0.2025 & -63.2238 & 0.4184 & -64.4438 & 0.6342 & -54.7544 & 0.8500 & -63.0185 & 1.0625 & -61.8521 & 1.2750 & -60.4126 \\
\hline 0.2059 & -75.0828 & 0.4217 & -58.8795 & 0.6375 & -69.1537 & 0.8533 & -90.7922 & 1.0658 & -58.1641 & 1.2783 & -60.0369 \\
\hline 0.2092 & -60.8279 & 0.4250 & -67.3196 & 0.6408 & -63.0661 & 0.8566 & -68.7794 & 1.0691 & -60.8006 & 1.2816 & -57.3396 \\
\hline 0.2125 & -65.4016 & 0.4283 & -67.3859 & 0.6441 & -68.0638 & 0.8600 & -75.6093 & 1.0725 & -66.0229 & 1.2850 & -61.0038 \\
\hline 0.2158 & -62.4642 & 0.4316 & -54.0376 & 0.6475 & -62.3672 & 1.2949 & -64.5761 & 1.2916 & -71.2011 & 1.2883 & -71.0445 \\
\hline
\end{tabular}


Emeshili O. Joseph, Emmanuel Eronu, Evans Ashigwuike; An Algorithm for Spectrum Hole Detection using Convex Optimization And Tensor Analysis In Cognitive Radio Network. Journal of Biomedical Engineering and Medical Imaging, Volume 6, No 6, December (2019), pp 1-24

\subsection{Procedure for Realising Algorithm}

The Algorithm will follow a particular trend and procedure, the flowchart in figure 11 represents the flow process for the algorithm, the large spectrum data from a wideband will undergo various processes before the spectrum hole can be detected as shown in figure 11 .

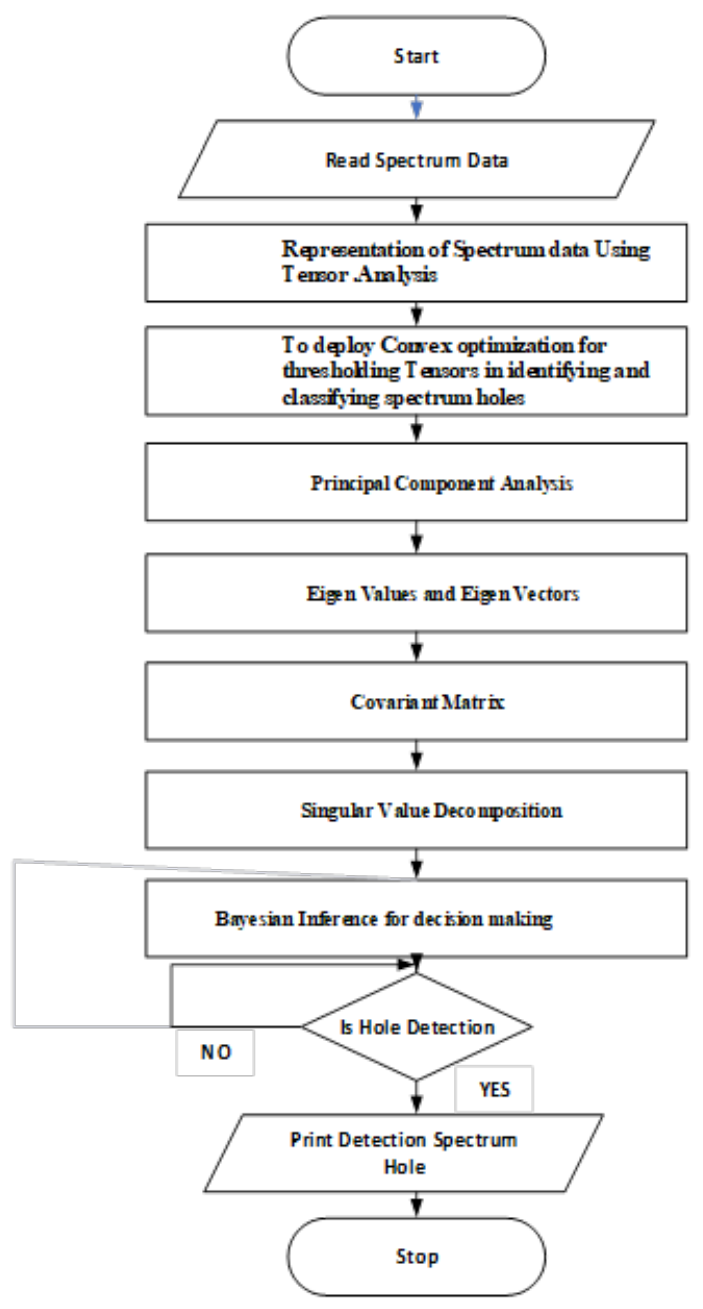

Figure 11 Flowchart showing the procedure for the algorithm 
When a constraint is introduced, the result will further narrow the search area, and this will further increase the possibility of detection as shown in figure 10 the red line is for thresholding and converging the search area.

The diagram in 12 is a single slice diagram while figure 12 is a combination of many slices which can be referred to as Tensor, this provides a unique representation for any instance of the frequency range,

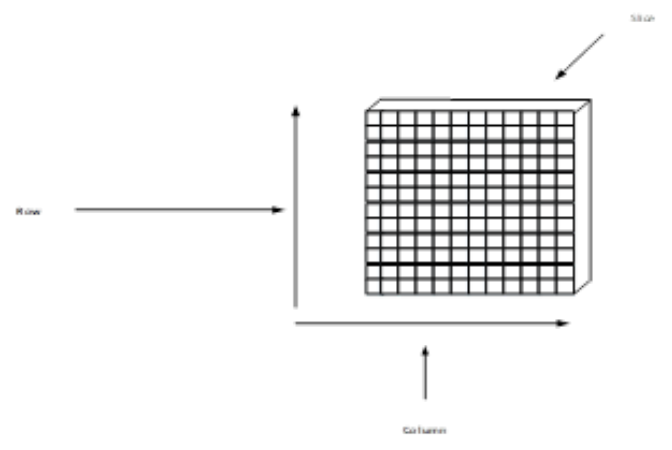

Figure 12 Diagram of a Front Slice

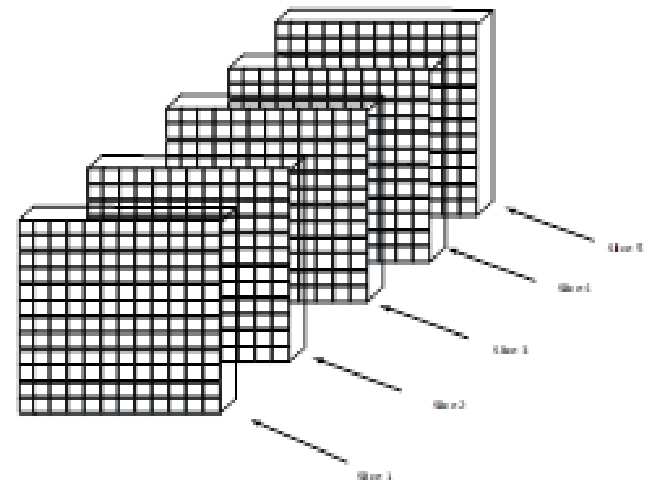

Figure 13 Several Slices with Hyperspaces with Row and Column

The space within the slice in figure 14 is called Hyperspace, and this has increased facility for frequency representation. In representing them, the tensors are defined based on a specific order
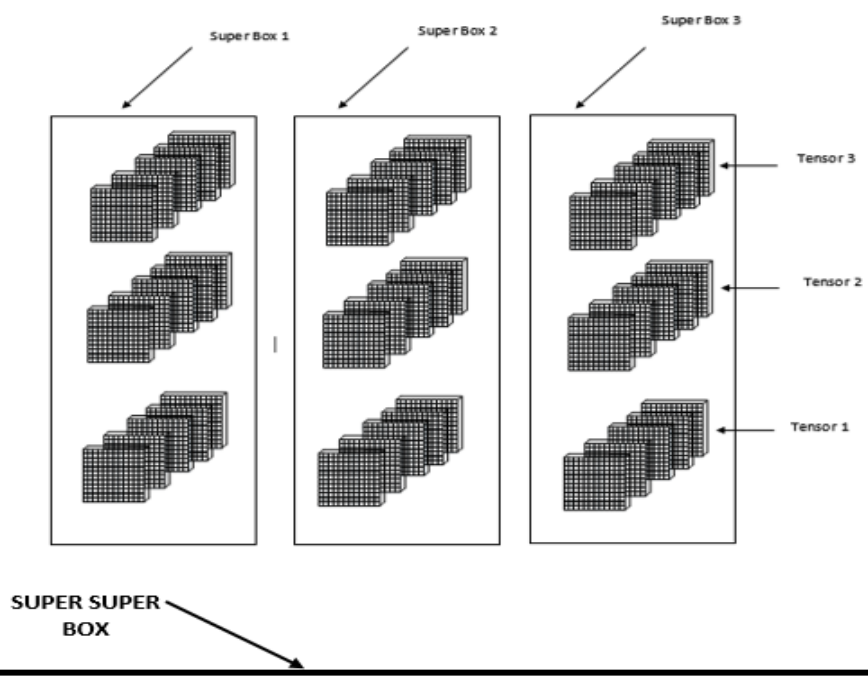

Figure 14 HyperSpace and Super Box 


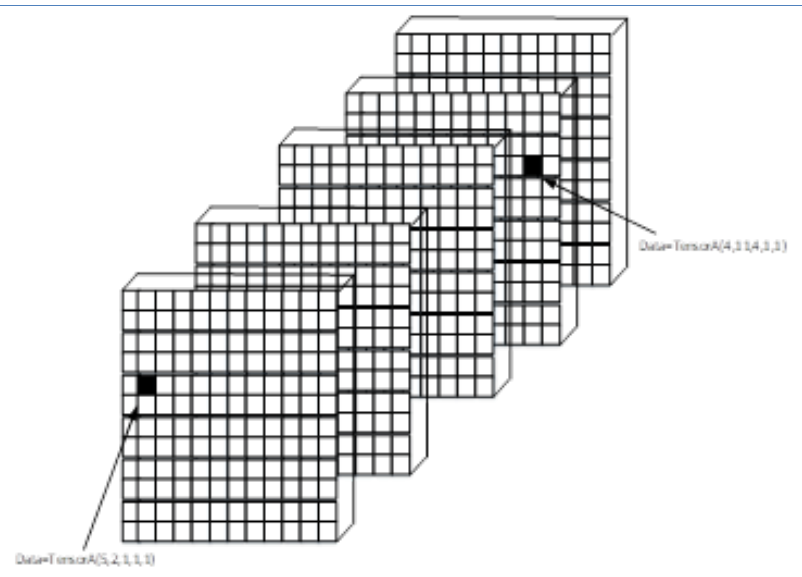

Figure 15 Tensor showing coordinate on Tensors

The demonstration here shows how the representation is done in tensor, the components for this are row,column,Slice,Tensor,Superbox. Figure 12, 13, 14 and 15 are graphical examples of the process of representing events using Tensors. Data command is given as follows

Data=TensorA(row,column,Slice,Tensor,Superbox)

Data=TensorA $(4,11,4,1,1)$

The command Tensor $A(4,11,4,1,1)$ represent various position

Data=TensorA $(5,2,1,1,1)$

Lets represent a spectrum data using random data.

$\mathrm{a}=\operatorname{randn}(1,5)$

$\mathrm{a}=\operatorname{randn}(1,5)$

$a=0.5377 \quad 1.8339 \quad-2.2588 \quad 0.8622 \quad 0.3188$

$b=\operatorname{randn}(1,5)$

$b=-1.3077 \quad-0.4336 \quad 0.3426 \quad 3.5784 \quad 2.7694$

$c=\operatorname{randn}(1,5)$

$c=-1.3499 \quad 3.0349 \quad 0.7254 \quad-0.0631 \quad 0.7147$

a covariant of all the spectrum data call be explored in matlab using this command

$a_{-} \operatorname{cov}=a^{*} . a^{\prime}$

a_cov $=a^{*}$. $a^{\prime}$

one major advantage of using covariant matrix is that it can transpose noise signal which cancel out completely. Exhibiting the command in matlab

$>$ a_cov $=a^{*} a^{\prime}$

a_cov $=9.5996$ 
A further extension of the data could generate $a$ random number 5 by 5 with the following command $a=$ randn $(5,5)$

This random number is assign to a as

$$
\begin{array}{rrrrrr}
a= & -0.2050 & 0.6715 & 1.0347 & 0.8884 & 1.4384 \\
-0.1241 & -1.2075 & 0.7269 & -1.1471 & 0.3252 \\
1.4897 & 0.7172 & -0.3034 & -1.0689 & -0.7549 \\
1.4090 & 1.6302 & 0.2939 & -0.8095 & 1.3703 \\
1.4172 & 0.4889 & -0.7873 & -2.9443 & -1.7115
\end{array}
$$

This method could be used for data for $b$ and $c$

$$
\begin{array}{lllll}
\begin{array}{l}
> \\
b=
\end{array}=\operatorname{randn}(5,5) & & & \\
-0.1022 & -0.0301 & -0.8637 & 1.5326 & -1.0891 \\
-0.2414 & -0.1649 & 0.0774 & -0.7697 & 0.0326 \\
0.3192 & 0.6277 & -1.2141 & 0.3714 & 0.5525 \\
0.3129 & 1.0933 & -1.1135 & -0.2256 & 1.1006 \\
-0.8649 & 1.1093 & -0.0068 & 1.1174 & 1.5442 \\
>>c=\operatorname{randn}(5,5) & & & \\
c= & & & & \\
0.0859 & -0.6156 & -1.4023 & 1.4193 & 0.6966 \\
-1.4916 & 0.7481 & -1.4224 & 0.2916 & 0.8351 \\
-0.7423 & -0.1924 & 0.4882 & 0.1978 & -0.2437 \\
-1.0616 & 0.8886 & -0.1774 & 1.5877 & 0.2157 \\
2.3505 & -0.7648 & -0.1961 & -0.8045 & -1.1658
\end{array}
$$

Exploring the tensor properties for infinite data representation, this is done by invoking the tensor command in Matlab below zero is assigned to tensor data.

Exploring the tensor properties of for infinite data representation, by invoking the tensor command in Matlab below zero is assigned to tensor data Considering a tensor of order $(5,5,7)$

> data_tensor $=$ zeros $(5,5,7)$

Initialising the first slice of the tensor 1 to zero

$$
\begin{aligned}
& \text { data_tensor(:,:,1) = } \\
& \begin{array}{lllll}
0 & 0 & 0 & 0 & 0
\end{array} \\
& \begin{array}{lllll}
0 & 0 & 0 & 0 & 0
\end{array} \\
& \begin{array}{lllll}
0 & 0 & 0 & 0 & 0
\end{array}
\end{aligned}
$$


Emeshili O. Joseph, Emmanuel Eronu, Evans Ashigwuike; An Algorithm for Spectrum Hole Detection using Convex Optimization And Tensor Analysis In Cognitive Radio Network. Journal of Biomedical Engineering and Medical Imaging, Volume 6, No 6, December (2019), pp 1-24

$\begin{array}{lllll}0 & 0 & 0 & 0 & 0\end{array}$

$\begin{array}{lllll}0 & 0 & 0 & 0 & 0\end{array}$

Initialising the first slice of the tensor 2 to zero

data_tensor(:,:,2) =

$\begin{array}{lllll}0 & 0 & 0 & 0 & 0\end{array}$

$\begin{array}{lllll}0 & 0 & 0 & 0 & 0\end{array}$

$\begin{array}{lllll}0 & 0 & 0 & 0 & 0\end{array}$

$\begin{array}{lllll}0 & 0 & 0 & 0 & 0\end{array}$

$\begin{array}{lllll}0 & 0 & 0 & 0 & 0\end{array}$

Initialising the first slice of the tensor 3 to zero

data_tensor(:,:,3) =

$\begin{array}{lllll}0 & 0 & 0 & 0 & 0\end{array}$

$\begin{array}{lllll}0 & 0 & 0 & 0 & 0\end{array}$

$\begin{array}{lllll}0 & 0 & 0 & 0 & 0\end{array}$

$\begin{array}{lllll}0 & 0 & 0 & 0 & 0\end{array}$

$\begin{array}{lllll}0 & 0 & 0 & 0 & 0\end{array}$

Initialising the first slice of the tensor 4 to zero

data_tensor(:,:,4) =

$\begin{array}{lllll}0 & 0 & 0 & 0 & 0\end{array}$

$\begin{array}{lllll}0 & 0 & 0 & 0 & 0\end{array}$

$\begin{array}{lllll}0 & 0 & 0 & 0 & 0\end{array}$

$\begin{array}{lllll}0 & 0 & 0 & 0 & 0\end{array}$

$\begin{array}{lllll}0 & 0 & 0 & 0 & 0\end{array}$

Initialising the first slice of the tensor 5 to zero

$\begin{array}{ccccc}\text { data_tensor(:,:,5) }= \\ 0 & 0 & 0 & 0 & 0 \\ 0 & 0 & 0 & 0 & 0 \\ 0 & 0 & 0 & 0 & 0 \\ 0 & 0 & 0 & 0 & 0 \\ 0 & 0 & 0 & 0 & 0\end{array}$

Initialising the first slice of the tensor 6 to zero

data_tensor(:,:,6) =

$\begin{array}{lllll}0 & 0 & 0 & 0 & 0\end{array}$

$\begin{array}{lllll}0 & 0 & 0 & 0 & 0\end{array}$

$\begin{array}{lllll}0 & 0 & 0 & 0 & 0\end{array}$

$\begin{array}{lllll}0 & 0 & 0 & 0 & 0\end{array}$

$\begin{array}{lllll}0 & 0 & 0 & 0 & 0\end{array}$

Initialising the first slice of the tensor 7 to zero 


$\begin{array}{ccccc}\text { data_tensor(:,:,7) }= \\ 0 & 0 & 0 & 0 & 0 \\ 0 & 0 & 0 & 0 & 0 \\ 0 & 0 & 0 & 0 & 0 \\ 0 & 0 & 0 & 0 & 0 \\ 0 & 0 & 0 & 0 & 0\end{array}$

Assigning Slice 1 data to a, using the command below

$$
\begin{aligned}
& \text { > data_tensor(:,:,1)=a } \\
& \text { data_tensor(:,:,1)= } \\
& \begin{array}{lllll}
0.2050 & 0.6715 & 1.0347 & 0.8884 & 1.4384 \\
-0.1241 & -1.2075 & 0.7269 & -1.1471 & 0.3252 \\
1.4897 & 0.7172 & -0.3034 & -1.0689 & -0.7549 \\
1.4090 & 1.6302 & 0.2939 & -0.8095 & 1.3703 \\
1.4172 & 0.4889 & -0.7873 & -2.9443 & -1.7115
\end{array}
\end{aligned}
$$

Assigning data to tensors slice 1

$$
\begin{aligned}
& \text { > data_tensor(:,:,1) } \\
& \text { ans }= \\
& \begin{array}{llllll}
0.2050 & 0.6715 & 1.0347 & 0.8884 & 1.4384 \\
-0.1241 & -1.2075 & 0.7269 & -1.1471 & 0.3252 \\
1.4897 & 0.7172 & -0.3034 & -1.0689 & -0.7549 \\
1.4090 & 1.6302 & 0.2939 & -0.8095 & 1.3703 \\
1.4172 & 0.4889 & -0.7873 & -2.9443 & -1.7115
\end{array}
\end{aligned}
$$

Slice 2 is initialised with zeros

> data_tensor(:,:,2)
answer =
$\begin{array}{lllll}0 & 0 & 0 & 0 & 0 \\ 0 & 0 & 0 & 0 & 0 \\ 0 & 0 & 0 & 0 & 0 \\ 0 & 0 & 0 & 0 & 0 \\ 0 & 0 & 0 & 0 & 0\end{array}$

Slice 3 is assign to $b$ data

$$
\begin{aligned}
& \text { >> data_tensor(:,:,3) = b } \\
& \text { data_tensor(:,:,3) = } \\
& \begin{array}{lllll}
-0.1022 & -0.0301 & -0.8637 & 1.5326 & -1.0891
\end{array} \\
& \begin{array}{llllll}
-0.2414 & -0.1649 & 0.0774 & -0.7697 & 0.0326
\end{array} \\
& \begin{array}{lllll}
0.3192 & 0.6277 & -1.2141 & 0.3714 & 0.5525
\end{array} \\
& \begin{array}{llllll}
0.3129 & 1.0933 & -1.1135 & -0.2256 & 1.1006
\end{array} \\
& \begin{array}{llllll}
-0.8649 & 1.1093 & -0.0068 & 1.1174 & 1.5442
\end{array}
\end{aligned}
$$


Emeshili O. Joseph, Emmanuel Eronu, Evans Ashigwuike; An Algorithm for Spectrum Hole Detection using Convex Optimization And Tensor Analysis In Cognitive Radio Network. Journal of Biomedical Engineering and Medical Imaging, Volume 6, No 6, December (2019), pp 1-24

The tensor here shows that slice 4 is assigned zeros

$$
\begin{aligned}
& \text { data_tensor }(:,:, 4)= \\
& \begin{array}{lllll}
0 & 0 & 0 & 0 & 0
\end{array} \\
& \begin{array}{lllll}
0 & 0 & 0 & 0 & 0
\end{array} \\
& \begin{array}{lllll}
0 & 0 & 0 & 0 & 0
\end{array} \\
& \begin{array}{lllll}
0 & 0 & 0 & 0 & 0
\end{array} \\
& \begin{array}{lllll}
0 & 0 & 0 & 0 & 0
\end{array} \\
& \text { > data_tensor(:,:,3) }
\end{aligned}
$$

\section{Convex Optimization}

Convex optimization is a subfield of optimization that studies the problem of minimizing convex functions over convex sets. Convex optimization is used to split frequencies into sub-bands; this will help in directing one to the most likely position where the available spectrum hole exists, convex optimization will help minimize the time taken for detection. The convexity makes optimization easier than the general case since the local minimum must be a global minimum, and first-order conditions are sufficient conditions 
for optimality. The general mathematical programming problem can be formulated by determining the $\mathrm{n}$ variables

Minimize $f_{0} x$

$$
f_{0} x \text { represents the frequency and energy level }
$$

Subject to $f_{i}(x) \leq 0, i=1 \ldots \ldots . \quad(4) m$,

this is a condition for convex optimization

$$
h_{i}(x) \leq 0, \quad i=1 \ldots \ldots \ldots \ldots \ldots . p
$$

In problem formulation, convex optimization is used with $\mathrm{x}$ as the variable

$x=\left(x_{1} \ldots \ldots \ldots \ldots \ldots \ldots \ldots x_{n}\right)$.

That optimizes function for the variables under test is

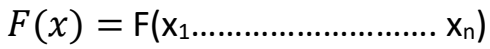

Which is called objective function, note that this is the constraint for optimization

$g(x)=g\left(x_{1} \ldots \ldots \ldots \ldots x_{n}\right) \geq 0 \quad i=1 \ldots \ldots \ldots \ldots \ldots \ldots . .1$

subject to linear and/or nonlinear inequality constraints

$$
g(x)=g\left(x_{1} \ldots \ldots \ldots \ldots x_{n}\right)=0 \quad i=l+1 \ldots \ldots \ldots \ldots m
$$

and to $\mathrm{m}-$ I linear and/or nonlinear equality constraint When the objective function and the $\mathrm{m}$ constraints are linear, the mathematical programming problem is a linear programming problem.

When either the objective function or one or more of the constraints are nonlinear, the programming problem is nonlinear. In considering the nonlinear programming problem we prefer always to think of a minimizing problem, but this implies no loss of generality since,

A generic optimisation problem (in minimisation form) is specified as

Min $\mathrm{f}_{0}(\mathrm{x})$ optimisation problem $f_{0}(x)=\leq 0 \quad i=1 \ldots \ldots \ldots m$

$\mathrm{h}_{1}(x)=0 \quad i=1 \ldots \ldots \ldots p$

The optimisation problem aims to find the $x$ that minimises

$\mathrm{f}_{0}(\mathrm{x})$ while satisfying $f_{0}(x)=\leq 0 \quad i=1 \ldots \ldots \ldots m$ and $\mathrm{h}_{1}(x)=0 \quad i=1 \ldots \ldots \ldots p$

The variable $x \in R$ is called the optimisation variable and the function $f 0: R^{n} \rightarrow R$

is known as the objective function.

The inequalities $f_{1}(x)=\leq 0$, are referred to as inequality constraints and the functions,

$f_{0}: R^{n} \longrightarrow$. are called inequality constraint functions.

The equalities $\mathrm{hi}(\mathrm{x})=0$ are called equality constraints and the functions $\mathrm{hi}(\mathrm{x}): \mathrm{R}^{\mathrm{n}} \longrightarrow \mathrm{R}$

$\mathrm{R}$ are known as equality constraint functions.

The domain $\mathrm{D}$ of the optimisation problem is the set of points for which the 
Emeshili O. Joseph, Emmanuel Eronu, Evans Ashigwuike; An Algorithm for Spectrum Hole Detection using Convex Optimization And Tensor Analysis In Cognitive Radio Network. Journal of Biomedical Engineering and Medical Imaging, Volume 6, No 6, December (2019), pp 1-24

objective and constraint functions are defined. A point $x \in D \times 2 D$ is called a feasible point if all constraints are satisfied. The optimisation problem is feasible if there is at least one feasible point.

A feasible solution $x^{*}$ is called a globally optimum solution if $f_{0}\left(x^{*}\right)=\leq f_{0}(x)$.

For all feasible $\mathrm{x}$, A feasible solution $\mathrm{x}$ is called a locally optimum solution if there exists an $\varepsilon>0$ such that $f_{0}\left(x^{*}\right)=\leq f_{0}(x)$ for all feasible $x$ that satisfies. $\left\|\mathrm{x}-\mathrm{x}^{*}\right\|_{2} \leq \varepsilon$

A fundamental property of convex optimisation problems is that any locally optimal point is also globally optimal.

This work will adopt the front slice technique since it works with the property of matrix and for easy representation, Figure 16 is a single slice which contains rows and column, this will help in describing the position of energy level against frequency within the block.

Frontal slice technique is used since it works with the property of matrix and for straightforward representation, as shown in figure 16.

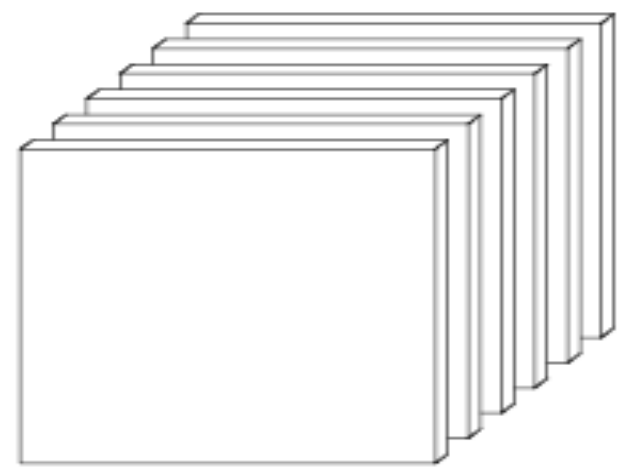

Figure 16 Various Tensor Slices and Fibres (Frontal slices: X::k (or Xk)

The inner product of two same-sized tensors is given below

$$
(x, y)=\sum_{i 1=1}^{I_{1}} \sum_{i 2=1}^{I_{2}} \ldots \ldots \ldots \sum_{i N=1}^{I_{N}} x_{i 1 i 2 \ldots \ldots i N} y_{i 1 i 2 \ldots \ldots i_{N}}
$$

\subsection{Principal Component Analysis}

The Spectrum data Principal component analysis (PCA) is a statistical procedure that uses an orthogonal transformation to convert a set of data acquired from the spectrum taking into account observations of possibly correlated variables (entities each of which takes on various numerical values) into a set of values of linearly uncorrelated variables called principal components. If there are $n$ observations with $p$ variables, then the number of distinct principal components is $\min (n-1, p)$.

This transformation is defined in such a way that the first principal component has the most significant possible variance (that is, it accounts for as much of the variability in the data as possible), and each succeeding component, in turn, has the highest variance possible under the constraint that it is orthogonal to the preceding components. The resulting vectors (each being a linear combination of the variables and 
containing $\mathrm{n}$ observations) are an uncorrelated orthogonal basis set. PCA is sensitive to the relative scaling of the original variables.

\subsection{Eigen Values \& Eigenvectors}

After using the method of Principal Component Analysis to locate the exact possible area where the hole could be located based on the pattern derived from the PCA to in linear algebra, an eigenvector or characteristic vector of a linear transformation is a non-zero vector that changes by only a scalar factor when that linear transformation is applied to it.

Let the Eigenvector represent a range of spectrum data, these stream of data on a plot will have a range of coefficients that will further describe their characteristics.

This stream of spectrum data could be represented as vector $\vec{v}$ while the vector is taken as $\lambda$

This means we could have a stream of data as

$$
\lambda=\lambda_{1}+\lambda_{2}+\lambda_{3}+\lambda_{4}+\cdots \ldots . \lambda_{n} \ldots \ldots
$$

Eigenvectors (for a square $m \times m$ matrix $\mathbf{S}$ ) $S \vec{v}=\lambda \vec{v}$

$$
A \vec{v}=\lambda \vec{v}
$$

Then we can conjugate to get $\underline{A \vec{v}}=\underline{\lambda \vec{v}}$

If the entries of $A$ are real, this becomes $A \underline{\vec{v}}=\underline{\lambda \vec{v}}$

This proves that complex eigenvalues of real-valued matrices come in conjugate pairs

Now transpose to get $\underline{\vec{v}}^{t} A^{T}=\underline{\vec{v}}^{t} \underline{\lambda}$. Because $\mathrm{A}$ is a symmetric matrix, we now have $\underline{\vec{v}}^{t} A=\underline{\vec{v}}^{t} \underline{\lambda}$

Multiply both sides of this equation on the right with $\vec{v}$, i.e. $\underline{\vec{v}}^{t} A \vec{v}=\underline{\vec{v}}^{t} \underline{\lambda} \vec{v}$

On the other hand multiply $A \vec{v}=\lambda \vec{v}$ on the left by $\underline{\vec{v}}^{t}$ to get $\underline{\vec{v}}^{t} A \vec{v}=\underline{\vec{v}}^{t} \lambda \vec{v}$

$\Rightarrow \underline{\vec{v}}^{t} \underline{\lambda} \vec{v}=\underline{\vec{v}}^{t} \lambda \vec{v} \Rightarrow \underline{\lambda}=\lambda \Rightarrow \lambda$ is real

0 If $\mathrm{A}$ is an $\mathrm{n} \times \mathrm{n}$ symmetric matrix, then any two eigenvectors that come from distinct eigenvalues are orthogonal. $\quad A \vec{v}_{i}=\lambda_{i} \vec{v}_{i}$

$>$ Left multiply with $\vec{v}_{j}^{T}$ to $A \vec{v}_{i}=\lambda_{i} \vec{v}_{i} \Rightarrow \vec{v}_{j}^{T} A \vec{v}_{i}=\vec{v}_{j}^{T} \lambda_{i} \vec{v}_{i}$

$>$ Similarly $\vec{v}_{i}^{T} A \vec{v}_{j}=\vec{v}_{i}^{T} \lambda_{j} \vec{v}_{j}$

$>$ From the above two equations $\left(\lambda_{j}-\lambda_{i}\right) \vec{v}_{i}^{T} \vec{v}_{j}=0 \quad \Rightarrow \vec{v}_{i}^{T} \vec{v}_{j}=0$

$\therefore \vec{v}_{j}$ and $\vec{v}_{i}$ are perpendicular, as also illustrated by the graph in figure 17 .

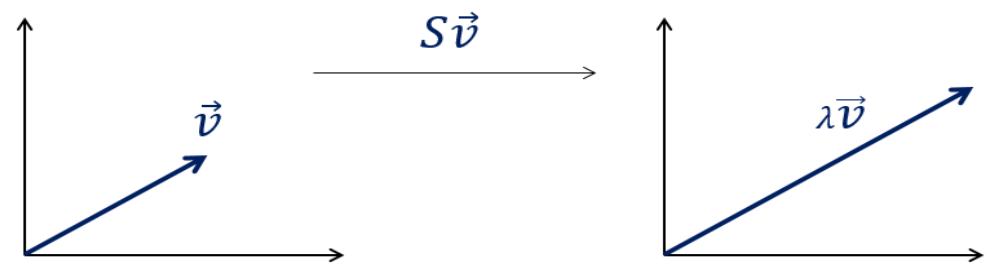

Figure 17 Eigen Value and Eigen Vector Representation 
Emeshili O. Joseph, Emmanuel Eronu, Evans Ashigwuike; An Algorithm for Spectrum Hole Detection using Convex Optimization And Tensor Analysis In Cognitive Radio Network. Journal of Biomedical Engineering and Medical Imaging, Volume 6, No 6, December (2019), pp $1-24$

$\operatorname{Variance}(\mathrm{x})=\frac{1}{n} \sum_{i=1}^{n} \quad\left(x_{i}-\underline{x}\right)^{2}$

\subsection{Eigen Values \& Eigenvectors Application}

Exploiting the properties Eigenvalues of real symmetric matrices which are real, Eigenvalues will further help to simplify the process by finding the root of the complex function, and the spectrum data is considered the vector $\vec{v}$ in this case

$$
A \vec{v}=\lambda \vec{v}
$$

Then we can conjugate to get $\underline{A \vec{v}}=\underline{\lambda \vec{v}} 7$

If the entries of $A$ are real, this becomes $A \underline{\vec{v}}=\underline{\lambda \vec{v}}$

This proves that complex eigenvalues of real-valued matrices come in conjugate pairs

Now transpose to get $\underline{\vec{v}}^{t} A^{T}=\underline{\vec{v}}^{t} \underline{\lambda}$. Because $\mathrm{A}$ is a symmetric matrix, we now have $\underline{\vec{v}}^{t} A=\underline{\vec{v}}^{t} \underline{\lambda}$

Multiply both sides of this equation on the right with $\vec{v}$, i.e. $\underline{\vec{v}}^{t} A \vec{v}=\underline{\vec{v}}^{t} \underline{\lambda} \vec{v}$

On the other hand multiply $A \vec{v}=\lambda \vec{v}$ on the left by $\underline{\vec{v}}^{t}$ to get $\underline{\vec{v}}^{t} A \vec{v}=\underline{\vec{v}}^{t} \lambda \vec{v}$

$$
\Rightarrow \underline{\vec{v}}^{t} \underline{\lambda} \vec{v}=\underline{\vec{v}}^{t} \lambda \vec{v} \Rightarrow \underline{\lambda}=\lambda \Rightarrow \lambda \text { is real }
$$

If $A$ is an $n \times n$ symmetric matrix, then any two eigenvectors that come from distinct eigenvalues are orthogonal.

$$
A \vec{v}_{i}=\lambda_{i} \vec{v}_{i}
$$

Left multiply with $\vec{v}_{j}^{T}$ to $A \vec{v}_{i}=\lambda_{i} \vec{v}_{i} \Rightarrow \vec{v}_{j}^{T} A \vec{v}_{i}=\vec{v}_{j}^{T} \lambda_{i} \vec{v}_{i}$

Similarly $\vec{v}_{i}^{T} A \vec{v}_{j}=\vec{v}_{i}^{T} \lambda_{j} \vec{v}_{j}$

From the above two equations $\left(\lambda_{j}-\lambda_{i}\right) \vec{v}_{i}^{T} \vec{v}_{j}=0$

$$
\Longrightarrow \vec{v}_{i}^{T} \vec{v}_{j}=0
$$

$\therefore \vec{v}_{j}$ and $\vec{v}_{i}$ are perpendicular

\subsection{Covariance Matrix}

Covariance Matrix will be used in explicitly detecting hole location in the spectrum, and this is after all the convergence has been realised.

Employing a Power spectral Density map (PSD), the general representation of a covariance is given in equation 7 below

$$
\text { Covariance }(x, y)=\frac{1}{n} \sum_{i=1}^{n} \quad\left(x_{i}-\underline{x}\right)\left(y_{i}-\underline{y}\right)
$$

- Covariance $(x, x)=\operatorname{var}(x)$

* Covariance $(x, y)=$ Covariance $(y, x)$

* $\operatorname{Covariance}(x, y)=\frac{1}{n} \sum_{i=1}^{n} \quad\left(x_{i}-\underline{x}\right)\left(y_{i}-\underline{y}\right)$

Lets assume a spectral Data plot in figure 18,19, 20 and 21, note that y axis represent the Energy level while the $x$ axis represent the frequency. 


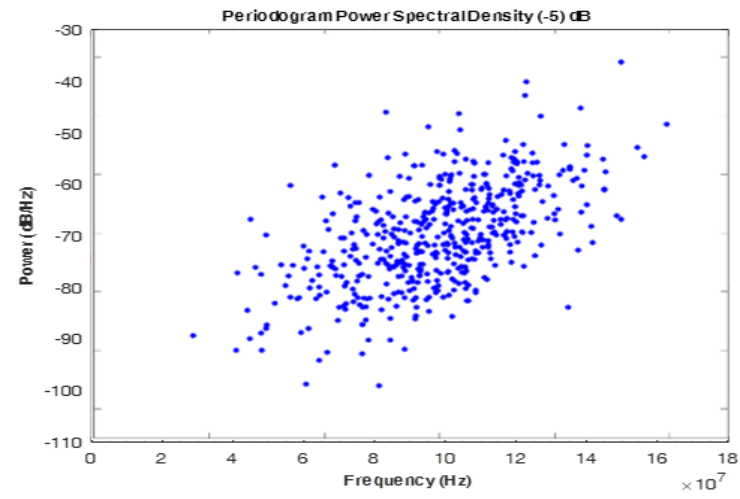

Figure 18 Plot showing Spectrum data

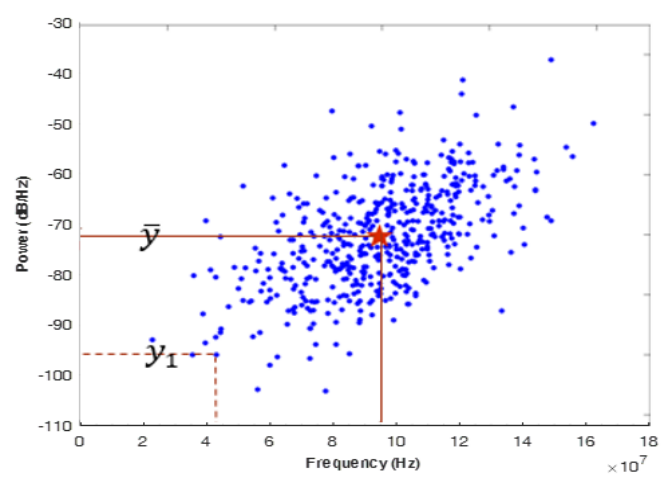

Figure 3.19 Point of Likelihood

The plot in figures 18,19, 20 and 21 show a scattered graph of spectrum data, and this further shows how hole location can be extracted, this platform will make it possible for covariance method to locate a point with a high possibility of a spectrum hole.

In Figure 3,18, the location for the spectrum hole using the relativity from the mean.

$$
\begin{gathered}
\operatorname{Covariance}(x, y)=\frac{1}{n} \sum_{i=1}^{n}\left(x_{i}-\underline{x}\right)\left(y_{i}-\underline{y}\right) \\
\operatorname{Covariance}(x, y)=\frac{1}{n} \sum_{i=1}^{n} \quad\left(x_{i}-\underline{x}\right)\left(y_{i}-\underline{y}\right)
\end{gathered}
$$

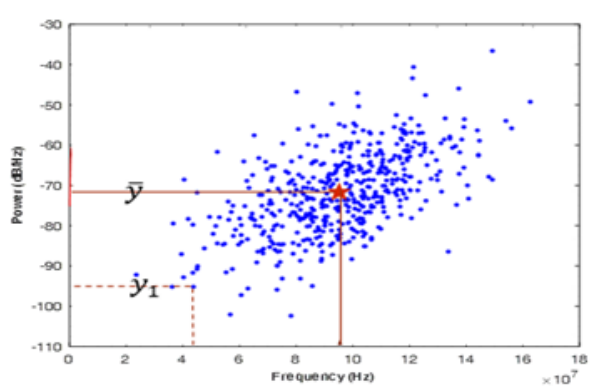

Figure 3.20 Calculating the mean in Covariance

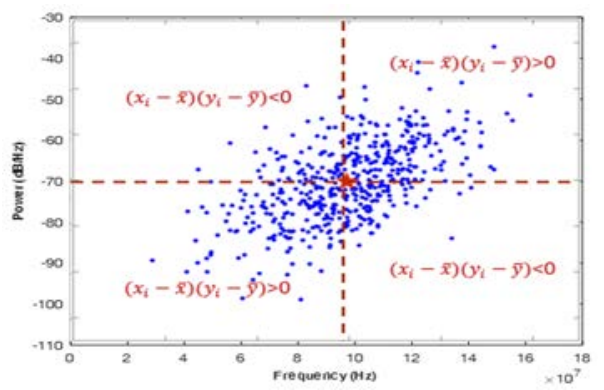

Figure 3.21 Extracting feature using Covariance method

$$
\begin{gathered}
\operatorname{Cov}(\Sigma)=\left[\operatorname{cov}\left(x_{1}, x_{1}\right) \operatorname{cov}\left(x_{1}, x_{2}\right) \cdots \operatorname{cov}\left(x_{1}, x_{m}\right) \operatorname{cov}\left(x_{2}, x_{1}\right) \operatorname{cov}\left(x_{2}, x_{2}\right) \cdots \operatorname{cov}\left(x_{2}, x_{m}\right)\right. \\
\left.\vdots \operatorname{cov}\left(x_{m}, x_{1}\right) \quad \vdots \operatorname{cov}\left(x_{m}, x_{2}\right) \quad \vdots \cdots \quad \cdots \operatorname{cov}\left(x_{m}, x_{m}\right)\right] \\
\operatorname{Cov}(\Sigma)=\frac{1}{n}(X-\underline{X})(X-\underline{X})^{T} ; \text { where } X=\left[\begin{array}{lll}
x_{1} x_{2} & \vdots x_{m}
\end{array}\right]
\end{gathered}
$$

\section{Conclusion}

This work came up with a procedure that uses Convex Optimization for Spectrum Hole Detection in Cognitive Radio Network Using Tensor Analysis. It explores the multi-variant capability of tensor and convex optimization Scheme for efficient and adaptive thresholding in identifying and classifying spectrum holes from the concept of tensors which will be used in spectrum hole identification. Other corresponding techniques were also explored like Principal Component Analysis, Eigen Value and Eigen Vector, with 
Emeshili O. Joseph, Emmanuel Eronu, Evans Ashigwuike; An Algorithm for Spectrum Hole Detection using Convex Optimization And Tensor Analysis In Cognitive Radio Network. Journal of Biomedical Engineering and Medical Imaging, Volume 6, No 6, December (2019), pp 1-24

these Speed of spectrum identification, will be improved upon based on the convergence technique to be adopted

Accuracy, which is a vital issue in spectrum hole detection, will be enhanced by advancing the use of the above-listed techniques in detection of spectrum holes. These techniques will then be improved upon via convex optimization in order to increase the speed and accuracy of decision making

\section{REFERENCES}

[1] B. R. J. J. M. Jaison Jacob, "Spectrum Prediction In Cognitive Radio Networks : A Bayesian Approach," in Eighth International Conference on Next Generation Mobile Applications, Services and Technology, 2014.

[2] M. K. Nawel Benghabrit, "Optimizing the Capacity Of Cognitive Radio Networks With Power Control And Variable Spectrum Allocation," Transport and Telecommunication, vol. 19, no. 2, p. 128-139, 2018.

[3] R. S. R. M. S. Raghave, "Continuous Wavelet Transform Based Spectrum Sensing in Cognitive Radio," Research Journal of Applied Sciences, Engineering and Technology, vol. 7, no. 5, pp. 986-988, 2018.

[4] W. Ahmed, MS Systems Eng. Centre For Telecommunications And Micro-Electronics, Faculty Of Health, Engineering And Science,Victoria University: Engineering And Science,Victoria University, 2010.

[5] S. A. Nadir Hussin, "United Arab Emirates University Scholarworks@UAEU," Spectrum Sharing In Cognitive Radio Networks With Quality Of Service Awareness , 2011.

[6] P. A. T. D. M. D. A. R. a. C. S. H. McHenry M. A., "Proceedings of the First International Workshop on Technology and Policy for Accessing Spectrum, ser. TAPAS '06. ACM..," in Accessing Spectrum, ser. TAPAS '06. New York, NY, USA: ACM,. [Online]. , New York, NY, USA: , 2006.

[7] A. Wisniewska, "A white paper on spectrum sharing," (2012).

[8] Y. F. ,. M. Adeel Ahmed, "Noise Variance Estimation for Spectrum Sensing in Cognitive Radio Networks," in Conference on Circuit and Signal Processing (CSP 2014), AASRI, 2014.

[9] T. a. Huseyin, " A Survey of Spectrum Sensing Algorithms for Cognitive Radio Applications," , IEEE communications surveys \& tutorials, vol. 11, no. 1, 2009.

[10] H. A. Tevfik Yucek, "A Survey of Spectrum Sensing Algorithms for Cognitive radio Application," IEEE Communication Survey and Tutorials, vol. 11, no. 1, (2009), .

[11] R. S. R. M. S. Raghave, "Continuous Wavelet Transform Based Spectrum Sensing in Cognitive Radio," Research Journal of Applied Sciences, Engineering and Technology, vol. 7, no. 5, pp. 986-988,, 2014.

[12] H. R. Mohammed Mehdi Saleh, "Quick Detection and Assignment of Spectrum Hole in Cognitive Radio," in International conference on Intelligent Systems, Data Mining and Information Technology (ICIDIT'2014), 2014.

[13] S. A. V. a. C. T. Xiaowen Gong, "Joint bandwidth and power allocation in wireless multi-user decode-andforward relay networks," in Conference: Proceedings of the IEEE International Conference on Acoustics, Speech, and Signal Processing, dallas, 2014. 
[14] B. E. C. G. F. M. d. A. Luiz Paulo de A., "Power Spectrum Detection Using Clustering, Simpo' Sio Brasileiro De Telecomunicac, O Es E Processamento De Sinais - Sbrt2017, 3-6 De Setembwro De 2017, Sa O Pedro, Sp," in De Telecomunicac, O Es E Processamento De Sinais - Sbrt2017, 3-6 De Setembwro De , Sa O Pedro, Sp, 2017.

[15] M. S. Y. S. E. A. Mohamed Shalaby, "Enhancement of Geometry and Throughput in LTE Femtocells Cognitive Radio Networks.," Wireless Personal Communications , vol. 77, no. 1, pp. 649-659 , 2014.

[16] M. K. Nawel Benghabrit1, "Optimizing the Capacity Of Cognitive Radio Networks With Power Control And Variable Spectrum Allocation," Transport and Telecommunication, vol. 19, no. 2, p. 128-139, 2018.

[17] d. B. Suseela, "energy based spectrum sensing, power spectrum estimation and papr analysis for cognitive radio networks," journal of theoretical and applied information technology, vol. 64, no. 3, 2018.

[18] N. Islam, Tensor and their application, New Delhi : new age international Publisher, 2006. 